\title{
Coleta de dados sobre o usuário do produto de vestuário: identificação de técnicas e ferramentas
}

\section{Data collect over the apparel product user: identification of techniques and tools}

\author{
Elen Makara, Instituto Federal de Educação, Ciência e Tecnologia de Santa \\ Catarina/Universidade Federal de Santa Catarina \\ makaraelen@gmail.com
}

\author{
Giselle S. A. D. Merino, Universidade Federal de Santa Catarina/Universidade do \\ Estado de Santa Catarina \\ gisellemerino@gmail.com
}

\begin{abstract}
Resumo
O processo de desenvolvimento de produto de vestuário requer a realização de diferentes pesquisas a respeito do produto, do contexto de uso e do usuário. Assim, no decorrer do processo, destaca-se a grande quantidade de informações, porém pesquisadores pontuam que a pesquisa sobre o usuário, é muitas vezes desconsiderada ou pouco explorada em ferramentas e métodos da área do Design de Moda. O objetivo deste artigo é identificar as técnicas e ferramentas utilizadas na coleta de dados sobre o usuário no processo de desenvolvimento de produtos de vestuário. Como procedimento metodológico foi realizada uma revisão sistemática da literatura, em 4 bases de dados, nas quais foram selecionadas 13 pesquisas, sendo tese e dissertações nacionais, e artigos científicos internacionais. Como resultado apresenta-se 10 modos para a coleta de dados, que são utilizados com maior frequência na fase de planejamento. Conclui-se que a coleta de dados sobre o usuário deve ser priorizada, e considerada em todas as fases do projeto, de modo a desenvolver produtos adequados aos mesmos.
\end{abstract}

Palavras-chave: Design de Moda, Desenvolvimento de Produto do Vestuário, Usuário.

\begin{abstract}
The clothing product development process requires different researches about the product, the context of use and the user. Thus, in the course of the process, a large amount of information stands out, but researchers point out that research on the user is disregarded or little explored in tools and methods in the area of Fashion Design. The purpose of this article is to identify the techniques and tools used to collect data about the user in the process of developing clothing products. As a methodological procedure, a systematic review of the literature was carried out in 4 databases, where 13 researches were selected, being thesis, dissertations, and scientific articles. As a result, there are 10 ways to collect data that are used most often in the planning phase. In conclusion, the collection of data about the user must be prioritized, and considered in all phases of the project, in order to develop products suitable for him.
\end{abstract}

Keywords: Fashion Design, Apparel Product Development, User.

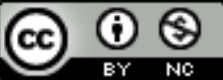




\section{Introdução}

O processo de desenvolvimento de produto de moda requer a realização de diferentes pesquisas a respeito do produto, do contexto de uso e do usuário. Cita-se como exemplos a pesquisa de mercado, de tema de coleção, de tendências, de materiais e cores, sobre o públicoalvo, análise de produtos concorrentes etc. Assim, como é possível perceber, o número de informações levantadas é grande, e por vezes, algumas delas acabam sendo desconsideradas no processo.

O produto de moda, que no caso desta pesquisa delimita-se a tratar do vestuário, possui um curto ciclo de vida visto que muda a cada estação do ano, e sofre influência do comportamento e estilo de vida do consumidor. Ou seja, para o seu desenvolvimento, além do levantamento de dados a respeito do próprio produto de vestuário e do contexto de uso, "é preciso ter consciência efetiva sobre o perfil do usuário do produto em desenvolvimento, pois é este universo que fornecerá as diretrizes para o direcionamento e formulação do projeto" (SANCHES, 2008, p.298).

Para o desenvolvimento de produtos, seja em ambiente acadêmico ou em empresas, a primeira atividade a ser realizada é o levantamento das necessidades dos usuários, sendo que as demais atividades e tomadas de decisões que surgem no decorrer do processo, são decorrentes desse levantamento (BACK, 2008). Entretanto, por mais que se saiba que é necessário ter conhecimento do usuário do produto de vestuário, é comum encontrar na literatura da área do Design de Moda métodos de projeto que priorizam as questões relacionadas com a estética do produto, como evidenciar o tema da coleção, ou da utilização das tendências de moda, ao invés de considerar as necessidades dos usuários (COLLET, 2016; NAKAYAMA, 2016).

Neste contexto, acrescentam-se as observações empíricas desta pesquisadora e docente responsável pelas disciplinas de Projeto de Moda, nas quais se percebe a dificuldade dos discentes em levantar informações relevantes sobre o usuário do produto, analisá-las e transformá-las em requisitos do projeto. Trata-se de uma situação que exige atenção ao orientá-los nestes procedimentos de coleta de dados com o usuário do produto, visto o cenário de escassez de métodos de projeto de moda com abordagem centrada no usuário.

Assim, considerando que no decorrer do processo de desenvolvimento de produto de moda está a grande quantidade de informações que devem ser pesquisadas, e que entre elas, a pesquisa sobre o usuário é pouco explorada, questiona-se quais as técnicas, métodos ou ferramentas têm sido utilizados para a coleta de dados sobre o usuário no projeto de Design de Moda.

O objetivo deste artigo é identificar as técnicas e ferramentas utilizadas na coleta de dados sobre o usuário no processo de desenvolvimento de produtos de vestuário. Essa pesquisa justificase dada a importância da coleta de dados sobre o usuário do produto de vestuário no decorrer de toda a prática projetual, e devido à carência de estudos da área do Design de Moda com abordagem centrada no usuário.

\section{Procedimentos Metodológicos}

Essa pesquisa se classifica segundo a sua natureza como uma pesquisa básica, com relação aos seus objetivos é exploratória-descritiva, e quanto à abordagem do problema se caracteriza como pesquisa qualitativa. Sobre os procedimentos técnicos refere-se a uma pesquisa bibliográfica. 
A pesquisa bibliográfica foi organizada de acordo com o guia para revisão sistemática proposto por Ferenhof e Fernandes (2016), que se divide em três etapas:

\section{Etapa 1: Definição do protocolo de pesquisa}

a) Definição da estratégia de busca:

- Bases de Dados Nacionais: ("processo de desenvolvimento" OR "desenvolvimento de produto" OR "metodologia projetual") AND ("design de moda").

- Bases de Dados Internacionais: ("product development" OR "development process" OR "design method*" OR "design tool") AND ("fashion design").

b) Definição das Bases de Dados:

- Bases de Dados Nacionais:

Biblioteca Brasileira de Teses e Dissertações (BDTD) e Catálogo de Teses e Dissertações da CAPES: Selecionadas por apresentarem as teses e dissertações defendidas em diferentes instituições de ensino e programas de pós-graduação do país.

- Bases de Dados Internacionais:

Scopus: Selecionada por ser a maior base de dados de resumos e citações de literatura revisada por pares. Abrange as áreas de ciências sociais aplicadas que contemplam as pesquisas da área do Design de Moda.

Web of Knowlegde: Selecionada por apresentar aproximadamente 12.000 periódicos, permitindo acesso a referências e resumos em todas as áreas do conhecimento.

c) Organização das Bibliografias: Definição de critérios de inclusão ou exclusão:

- Recorte de tempo: últimos 6 anos - 2015 a 2021.

- Inclusão das pesquisas que continham título, resumo e/ou palavras-chave dentro da temática buscada;

- Remoção das referências duplicadas;

- Leitura das pesquisas disponíveis para download no período de realização da revisão sistemática.

2. Etapa 2: Análise dos Dados - Análise sistemática das pesquisas selecionadas para leitura na íntegra, que consiste na interpretação destas pesquisas sobre a temática pesquisada, a coleta de dados sobre o usuário no desenvolvimento do produto de vestuário.

3. Etapa 3: Síntese dos Resultados: Como resultado, identificou-se nas pesquisas selecionadas na revisão sistemática, o modo (técnica, ferramenta, método, equipamento, etc.) como cada uma realizou a coleta de dados sobre o usuário.

\section{Revisão Sistemática}

Esta revisão sistemática foi realizada em 4 bases de dados, sendo 2 nacionais, a BDTD e o Catálogo de Teses e Dissertações da CAPES, e duas internacionais, a Scopus e Web of Knowledge. Foi realizada entre os meses de outubro e novembro de 2019, e posteriormente atualizada, em março de 2021. A busca retornou um montante de 206 pesquisas, das quais, retirando as referências duplicadas, restaram 131 pesquisas para uma seleção a partir da leitura dos títulos e resumos. A partir destas leituras, foram selecionadas 59 pesquisas para a leitura dinâmica, entretanto 12 destas pesquisas não estavam disponíveis, restando apenas 47 pesquisas para leitura. 
Após a leitura dinâmica, foram selecionadas 13 pesquisas que estavam dentro da temática buscada, pois apresentaram pesquisas que abordam a coleta de dados sobre o usuário no decorrer do processo de desenvolvimento de produto de vestuário (Figura 1).

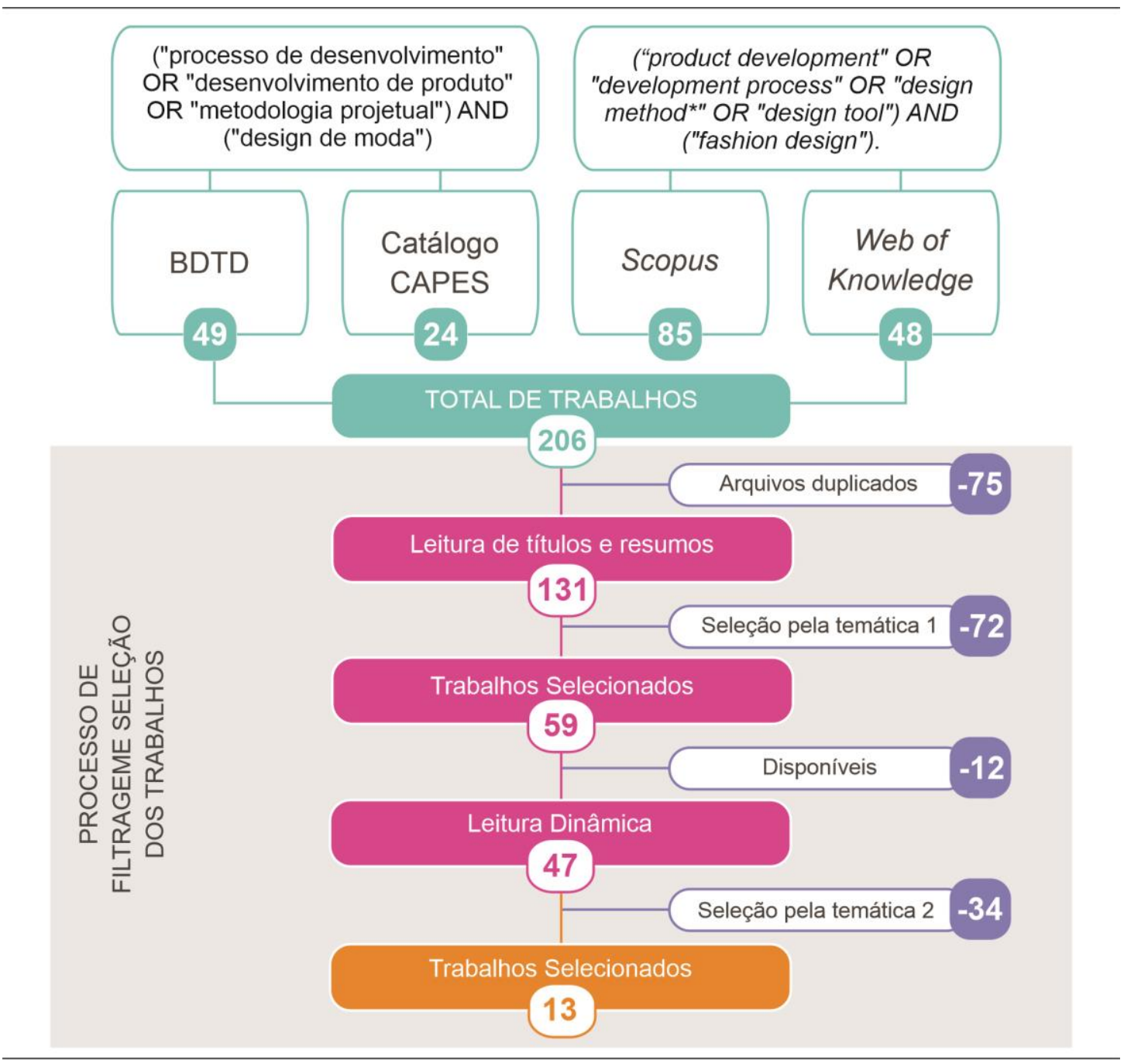

Figura 1: Síntese da Revisão Sistemática nas Bases de Dados e Pesquisas Selecionadas. Fonte: as autoras.

A Figura 2 apresenta as 13 pesquisas selecionadas, provenientes das bases de dados nacionais e internacionais, o nome dos autores, o ano da publicação, a instituição (pesquisas nacionais), a origem (pesquisas internacionais) o tipo de trabalho e o título da pesquisa. 


\begin{tabular}{|c|c|c|c|c|c|}
\hline & Autor & Ano & $\begin{array}{c}\text { Instituição/ } \\
\text { Origem }\end{array}$ & $\begin{array}{l}\text { Tipo de } \\
\text { Trabalho }\end{array}$ & Título \\
\hline \multirow{5}{*}{ 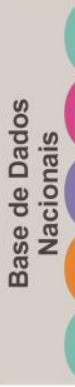 } & Collet & 2016 & UFRGS & Dissertação & $\begin{array}{l}\text { Método para a moda complementado por metodologias de design de produto: } \\
\text { Aplicação no desenvolvimento de produtos de moda praia para estomizadas. }\end{array}$ \\
\hline & Nakayama & 2016 & UFPE & Dissertação & $\begin{array}{l}\text { Desenvolvimento de produtos de moda para pessoas com mobilidade reduzida: } \\
\text { ferramenta metodológica pautada na ergonomia. }\end{array}$ \\
\hline & Souza & 2016 & USP & Dissertação & $\begin{array}{l}\text { Proposta de metodologia para adaptação de vestuário para pessoas com } \\
\text { deficiência física (cadeirante). }\end{array}$ \\
\hline & Mentone & 2018 & USP & Dissertação & $\begin{array}{l}\text { Proposta de desenvolvimento de produtos de malha para gestantes utilizando a } \\
\text { tecnologia seamless. }\end{array}$ \\
\hline & Brogin & 2019 & UFPR & Tese & $\begin{array}{l}\text { Método de Design para cocriação de moda funcional para pessoas com } \\
\text { deficiência. }\end{array}$ \\
\hline \multirow{8}{*}{ 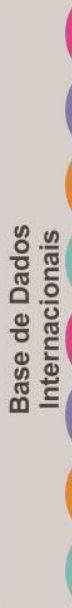 } & Lee et al. & 2015 & $\begin{array}{c}\text { China } \\
\text { Reino Unido }\end{array}$ & Artigo & Fuzzy association rule mining for fashion product development. \\
\hline & Tse e Chan & 2016 & China & Artigo & $\begin{array}{l}\text { New approach for fashion design: Case study of employing user-oriented } \\
\text { method to design mother-to-be party dress. }\end{array}$ \\
\hline & $\begin{array}{l}\text { Lü, Chen } \\
\text { e Sui }\end{array}$ & 2016 & China & Artigo & Event-related potentials technique using in affective fashion design. \\
\hline & Hong et al. & 2017 & $\begin{array}{l}\text { China } \\
\text { França }\end{array}$ & Artigo & $\begin{array}{l}\text { Virtual reality-based collaborative design method for designing customized } \\
\text { garment for disabled people with scoliosis. }\end{array}$ \\
\hline & Liu et al. & 2018 & $\begin{array}{l}\text { China } \\
\text { França }\end{array}$ & Artigo & Parametric design of garment flat based on body dimension. \\
\hline & $\begin{array}{l}\text { Nakayma e } \\
\text { Martins }\end{array}$ & 2018 & Brasil & Artigo & $\begin{array}{l}\text { Fashion Design Methodology Tools in Products' Development for People with } \\
\text { Disabilities and Low Mobility. }\end{array}$ \\
\hline & Liu et al. & 2019 & $\begin{array}{l}\text { China } \\
\text { França }\end{array}$ & Artigo & Parametric design of garment pattern based on body dimensions. \\
\hline & $\begin{array}{l}\text { Townsend } \\
\text { et al. }\end{array}$ & 2019 & Reino Unido & Artigo & $\begin{array}{l}\text { Fashioning clothing with and for mature women: a small-scale sustainable } \\
\text { design business model. }\end{array}$ \\
\hline
\end{tabular}

Figura 2: Pesquisas selecionadas na Revisão Sistemática. Fonte: as autoras.

Na sequência, descreve-se cada uma das pesquisas selecionadas na revisão sistemática de acordo com o objetivo, o usuário pesquisado, as informações levantadas e o modo utilizado para a coleta de dados sobre o usuário.

A pesquisa de Collet (2016) teve como objetivo desenvolver um método adaptado, a partir dos métodos propostos por autores da área do design e do design de moda, para a criação de produtos de moda inclusivos que captasse a real necessidade do usuário. Após a elaboração do método adaptado, este foi aplicado no desenvolvimento de uma coleção de moda praia para mulheres estomizadas. Para a aplicação, optou-se por realizar a coleta de dados sobre o usuário em fontes bibliográficas, a fim de evitar expectativas e não submeter pessoas desnecessariamente a qualquer tipo de experiência. Para a autora, o objetivo do trabalho foi o desenvolvimento do método, motivo pelo qual não foi apresentada, com detalhes, a aplicação, mas como resultado das roupas de moda praia desenvolvidas, tem-se que estas não comprometam o uso da bolsa coletora, colaboraram no cotidiano do usuário, e contribuíram física e psicologicamente nas atividades da vida social. A Figura 3 apresenta a síntese da pesquisa de Collet (2016), informa o objetivo, o usuário pesquisado, as informações levantadas, e a técnica utilizada. 


\begin{tabular}{c}
\hline \multicolumn{1}{c}{ Pesquisa de Collet (2016) } \\
\hline $\begin{array}{c}\text { Objetivo } \\
\text { adaptado para criação de } \\
\text { produtos de moda inclusivos }\end{array}$ \\
\hline $\begin{array}{c}\text { Informações Levantadas } \\
\text { - Bolsa não deve ficar aparente } \\
\text { de modo a causar } \\
\text { constrangimentos } \\
\text {-Roupa deve colaborar com o } \\
\text { cotidiano do usuário/com suas } \\
\text { atividades da vida social } \\
\text { - Roupa não deve comprometer o } \\
\text { uso da bolsa }\end{array}$ \\
\hline
\end{tabular}

Figura 3: Síntese da Pesquisa de Collet (2016). Fonte: as autoras.

A pesquisa de Nakayama (2016) teve como objetivo elaborar uma ferramenta metodológica direcionada ao ensino e a projeto de produtos de vestuário para pessoas com mobilidade reduzida. A partir da pesquisa bibliográfica a respeito de pessoas com mobilidade reduzida, a autora definiu requisitos para o projeto de moda e na sequência estabeleceu concordância com as diretrizes metodológicas de autores do design e design de moda. Assim obteve-se uma ferramenta com as fases e os requisitos projetuais para o projeto de produtos de moda para pessoas com mobilidade reduzida. A Figura 4 apresenta a síntese da pesquisa de Nakayama (2016), informa o objetivo, o usuário pesquisado, as informações levantadas, e a técnica utilizada.

\begin{tabular}{l|l}
\hline \multicolumn{1}{c}{ Pesquisa de Nakayama (2016) } \\
\hline \multicolumn{1}{c}{ Objetivo } & Usuário Pesquisado \\
\hline $\begin{array}{c}\text { Elaborar uma ferramenta } \\
\text { e ao projeto de produtos de moda, } \\
\text { especificamente vestuário, para } \\
\text { pessoas com mobilidade reduzida. }\end{array}$ & $\begin{array}{c}\text { Pessoas com mobilidade } \\
\text { reduzida }\end{array}$ \\
\hline \multicolumn{1}{|c|}{ Informações Levantadas } \\
- Delimitações do público - \\
conceituais e práticas \\
- Requisitos têxteis \\
- Requisitos de medidas \\
- Requisitos de modelagem \\
- Requisitos de costuras e \\
aviamentos \\
- Requisitos de avaliação e \\
finalização do produto \\
\hline
\end{tabular}

Figura 4: Síntese da Pesquisa de Nakayama (2016). Fonte: as autoras.

A pesquisa de Souza (2016) teve como objetivo propor uma metodologia para o desenvolvimento de vestuário adaptado para cadeirantes. A autora realizou um questionário com 
5 pacientes e 5 profissionais da área de saúde, a fim de verificar quais as necessidades e maiores dificuldades no ato de vestir e despir o vestuário. Na sequência, para definição dos tecidos, selecionou-se 11 amostras com as quais se realizou ensaios físicos (gramatura, espessura, tendência à formação de pilling ${ }^{1}$ e snagging ${ }^{2}$, determinação do alongamento e alteração dimensional, e gestão da umidade). Após análise dos testes físicos e do questionário, foram selecionados os 7 tecidos mais indicados para a confecção do vestuário para cadeirantes.

Como resultado, foram confeccionados 9 protótipos com adaptações que levam em consideração a facilidade do ato de vestir e despir, como modelagem das peças sem forro, sem bolso traseiro, bolsos funcionais na frente, cós com elástico, uso de aviamentos adequados como botões magnéticos e zíperes com puxador de argola. Após a confecção dos protótipos adaptados, realizou-se um teste de usabilidade com a calça jeans masculina, em que o tempo médio que o usuário levava para vestir-se, de 15 a 28 minutos, diminuiu para 5 minutos por causa das adaptações sugeridas. A Figura 5 apresenta a síntese da pesquisa de Souza (2016), informa o objetivo, o usuário pesquisado, as informações levantadas, e as técnicas utilizadas.

\begin{tabular}{|c|c|}
\hline \multicolumn{1}{c}{ Pesquisa de Souza (2016) } \\
\hline Objetivo & Usuário Pesquisado \\
\hline $\begin{array}{c}\text { Propor uma metodologia para o } \\
\text { desenvolvimento de vestuário } \\
\text { adaptado para pessoas com } \\
\text { deficiência física (cadeirantes) }\end{array}$ & $\begin{array}{c}\text { Cadeirantes } \\
\text { Profissionais da Saúde }\end{array}$ \\
\hline \multicolumn{1}{|l|}{ Informações Levantadas } \\
\hline $\begin{array}{l}\text { - Necessidades e dificuldades no } \\
\text { ato de vestir e despir o vestuário }\end{array}$ \\
\hline $\begin{array}{l}\text { - Funcionalidade da peça de } \\
\text { vestuário adaptada/Redução do } \\
\text { tempo que o cadeirante leva para } \\
\text { se vestir }\end{array}$ \\
\hline
\end{tabular}

Figura 5: Síntese da Pesquisa de Souza (2016). Fonte: as autoras.

A pesquisa de Mentone (2018) teve como objetivo desenvolver um protótipo de vestuário para gestantes, utilizando a tecnologia seamless (sem costura lateral). A autora realizou uma entrevista com 30 gestantes para verificar os desconfortos ocasionados pelas roupas durante a gestação. A partir das informações coletadas, identificou-se a necessidade de vestimentas que privilegiassem o conforto das mamas, e então foram desenvolvidos dois protótipos de vestuário com a tecnologia seamless. A partir dos protótipos, foram fabricadas 32 amostras em diferentes materiais, com as quais se realizou ensaios físicos (densidade linear, determinação de espessura, do alongamento e da elasticidade, tendência à formação de snagging, gestão da umidade e determinação da absorção por capilaridade) a fim de obter informação para a escolha do material adequado para o vestuário

\footnotetext{
${ }^{1}$ Tendência do tecido em apresentar bolinhas.

2 Tendência de enroscamento: quando as fibras e fios em tecidos são presos e puxados para longe da superfície dos tecidos.
} 
das gestantes. Também foi realizado um teste de usabilidade para verificar qual dos protótipos proporciona maior conforto e funcionalidade. Como resultado, um protótipo foi bem avaliado por não ter costuras que podem causar desconforto, por sustentar as mamas, e pelo tecido não reter umidade, já que gestantes têm maior produção de suor. Como sugestão de melhoria para este protótipo é ofertá-lo em cores escuras para não evidenciar os mamilos que ficam proeminentes na gestação. A Figura 6 apresenta a síntese da pesquisa de Mentone (2018), informa o objetivo, o usuário pesquisado, as informações levantadas, e as técnicas utilizadas.

\begin{tabular}{l|l}
\hline \multicolumn{1}{c}{ Pesquisa de Mentone (2018) } \\
\hline \multicolumn{1}{|c|}{ Objetivo } \\
\hline $\begin{array}{l}\text { Desenvolver um protótipo de } \\
\text { vestuário para gestantes utilizando } \\
\text { a tecnologia seamless. }\end{array}$ \\
\hline \multicolumn{1}{|c|}{ Informações Levantadas } \\
\hline $\begin{array}{l}\text { - Desconfortos ocasionados pelas } \\
\text { roupas no período da gestação; } \\
\text { - Necessidade de vestimentas que } \\
\text { privilegiassem conforto das mamas }\end{array}$ \\
$\begin{array}{l}\text { - Verificação Protótipo: maior } \\
\text { conforto e funcionalidade; melhor } \\
\text { sustentação das mamas; colabora } \\
\text { com a não retenção de umidade. }\end{array}$ \\
\hline
\end{tabular}

Figura 6: Síntese da Pesquisa de Mentone (2018). Fonte: as autoras.

A pesquisa de Brogin (2019, p.35) teve como objetivo "desenvolver, aplicar e avaliar um método de desenvolvimento de moda funcional para pessoas com deficiência motora, com base na cocriação". A autora propôs o método, que foi na sequência aplicado de quatro maneiras. Para este artigo, torna-se relevante considerar a aplicação do método realizada com os usuários reais, para analisar os modos de coleta de dados, que envolveu a aplicação de questionário com usuários e cuidadores; a análise da tarefa de vestir e despir, a análise de produtos concorrentes ou similares, aplicação do design colaborativo para criação do vestuário, tomada de medidas corporais, e prova de protótipos.

Como conclusão, os participantes avaliaram que os vestuários desenvolvidos a partir do método respondem às questões de ergonomia, usabilidade e conforto, proporcionando autonomia aos usuários e cuidadores, e ganho de tempo ao vestir e despir. A Figura 7 apresenta a síntese da pesquisa de Brogin (2019), informa o objetivo, o usuário pesquisado, as informações levantadas, e a técnica e/ou ferramentas utilizadas. 


\begin{tabular}{|c|c|}
\hline \multicolumn{2}{|c|}{ Pesquisa de Brogin (2019) } \\
\hline Objetivo & Usuário Pesquisado \\
\hline $\begin{array}{l}\text { Desenvolver, aplicar e avaliar um } \\
\text { método de desenvolvimento de } \\
\text { moda funcional para pessoas com } \\
\text { deficiência motora, com base na } \\
\text { cocriação }\end{array}$ & $\begin{array}{l}\text { - Pessoas com deficiência leve ou } \\
\text { moderada, e severa. } \\
\text { - Cuidadores (familiares) } \\
\text { - Terapeutas ocupacionais ou } \\
\text { fisioterapeutas }\end{array}$ \\
\hline Informações Levantadas & Técnica/Ferramenta Utilizada \\
\hline $\begin{array}{l}\text { - Necessidade de adaptação no } \\
\text { vestuário } \\
\text { - Modelos, acessórios, tecidos que } \\
\text { dificultam o vestir e despir } \\
\text { - Necessidade de auxilio ao vestir } \\
\text { e despir } \\
\text { - Peças que causam desconforto } \\
\text { ao vestir e despir }\end{array}$ & Questionário \\
\hline $\begin{array}{l}\text { - Dificuldades ao vestir/despir } \\
\text { peças de roupas } \\
\text { - Dificuldade com aviamento das } \\
\text { peças (ex.: botões e zíperes) }\end{array}$ & Análise da Tarefa \\
\hline $\begin{array}{l}\text { - Busca por soluções para o } \\
\text { vestuário }\end{array}$ & $\begin{array}{l}\text { Análise de Produtos } \\
\text { Concorrentes ou Similares }\end{array}$ \\
\hline $\begin{array}{l}\text { - Criação da peça, } \\
\text { - Escolha dos materiais adequados }\end{array}$ & Co-criação \\
\hline $\begin{array}{l}\text { - Medidas em diferentes posições } \\
\text { do usuário (ex.: sentado e deitado) }\end{array}$ & Medidas Corporais \\
\hline $\begin{array}{l}\text { - Verificar adequação da peça } \\
\text { - Facilidade ao vestir e despir }\end{array}$ & $\begin{array}{c}\text { Teste de Usabilidade } \\
\text { (Avaliação dos modelos) }\end{array}$ \\
\hline
\end{tabular}

Figura 7: Síntese da Pesquisa de Brogin (2019). Fonte: as autoras.

A pesquisa de Lee et al. (2015) teve como objetivo desenvolver uma ferramenta de mineração de dados com regras de associação difusa, denominada fuzzy association rule mining (FARM), de modo a auxiliar na eficiência e na eficácia do desenvolvimento de novos produtos (DNP) nas empresas de confecção fast-fashion. O uso da ferramenta FARM foi avaliado em uma empresa de moda sediada em Hong Kong pelo período de 6 meses. Entre os resultados observados a FARM proporcionou maior satisfação do cliente e redução do tempo de DNP, pois a ferramenta permite uma rápida identificação das necessidades e preferências dos clientes com relação aos diferentes estilos de vestuário. A Figura 8 apresenta a síntese da pesquisa de Lee et al. (2015), informa o objetivo, o usuário pesquisado, as informações levantadas, e a técnica e/ou ferramentas utilizadas. 


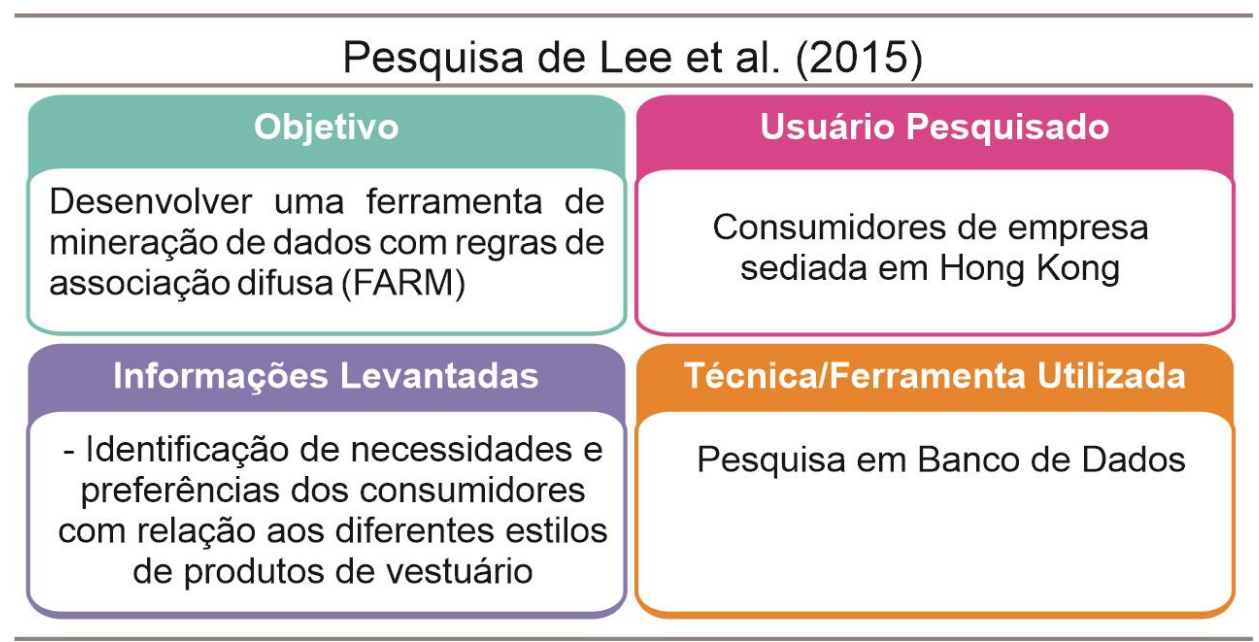

Figura 8: Síntese da Pesquisa de Lee et al (2015). Fonte: as autoras.

A pesquisa de Tse e Chan (2016) teve como objetivo estudar o uso dos métodos orientados ao usuário para desenvolver uma coleção de vestidos de festa para gestantes. Para isso foi realizada inicialmente uma entrevista com 4 mulheres, que compartilharam suas experiências e descreveram os requisitos para um vestido de festa para gestante. Após a análise dos dados, definiram-se seis requisitos, e com estes foi realizada uma nova pesquisa, com 18 gestantes, de modo a estabelecer a ordem de importância: 1) revelar a gravidez, 2) evitar constrangimentos, 3) seguro para se movimentar, 4) mostrar uma futura mãe feliz, 5) adicionar um toque de glamour, 6) coincidir com minha identidade social e profissional.

Tse e Chan (2016) exploraram o segundo requisito por meio de pesquisas de fotos na internet de gestantes, cujas roupas poderiam contribuir para algum tipo de constrangimento em jantares e eventos. Foram encontradas sete possíveis situações de constrangimentos, com as quais foi realizado um estudo com 10 grávidas que indicaram quais situações de constrangimentos é preferível evitar, obtendo a seguinte ordem: 1) vestido não encoste nos alimentos e bebidas, 2) vestido não derrube objetos, 3) vestido não encolha no comprimento, 4) que laços e transpasses não fiquem folgados, 5) não prenda o cabelo, 6) não pise na barra no vestido, 7) ao sentar a roupa permaneça igual. O desenvolvimento da coleção levou em consideração os dois requisitos mais importantes: revelar a gravidez e evitar constrangimentos, e como resultado foram apresentados seis looks orientados às necessidades das usuárias. A Figura 9 apresenta a síntese da pesquisa de Tse e Chan (2016), informa o objetivo, o usuário pesquisado, as informações levantadas, e a técnica e/ou ferramentas utilizadas. 


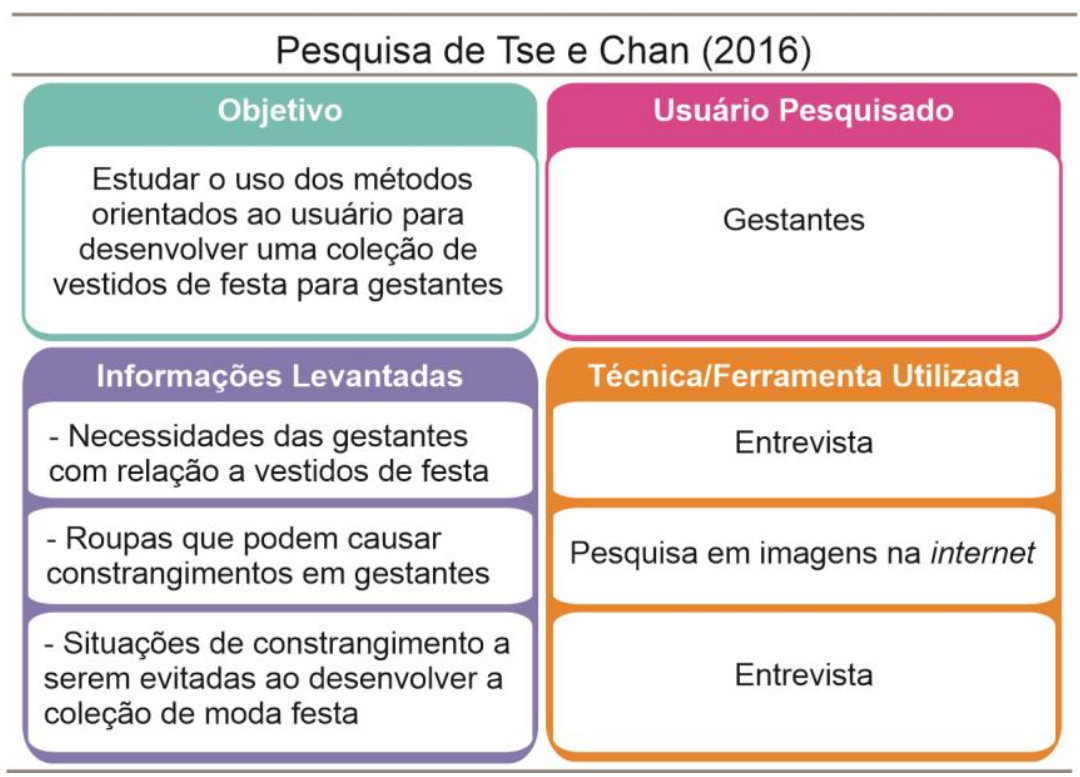

Figura 9: Síntese da Pesquisa de Tse e Chan (2016). Fonte: as autoras.

A pesquisa de Lü, Chen e Sui (2016) teve como objetivo propor um método de análise psicológica dos consumidores, com o qual seria possível fornecer dados para equipes de desenvolvimento de produtos, para que estes consigam atender às necessidades dos consumidores de maneira eficaz. O estudo analisa as respostas psicológicas dos consumidores quando estes eram expostos à imagem de peças de roupas. Assim, a preferência por determinados produtos de vestuário pode ser verificada, analisando as atividades cognitivas dos consumidores, por meio de ondas cerebrais. Entre as limitações do estudo está o fato de que a medição dos potenciais cerebrais exigem dispositivos específicos e ambientes de laboratório controlados. A Figura 10 apresenta a síntese da pesquisa de Lü, Chen e Sui (2016), informa o objetivo, o usuário pesquisado, as informações levantadas, e a técnica ou ferramentas utilizadas.

\begin{tabular}{|c|c|}
\hline \multicolumn{2}{|c|}{ Pesquisa de Lü, Chen e Sui (2016) } \\
\hline Objetivo & Usuário Pesquisado \\
\hline $\begin{array}{c}\text { Propor um método de análise } \\
\text { psicológica dos consumidores de } \\
\text { modo a fornecer dados para } \\
\text { equipes de desenvolvimento de } \\
\text { produtos }\end{array}$ & Usuário não especificado \\
\hline Informações Levantadas & Técnica/Ferramenta Utilizada \\
\hline $\begin{array}{c}\text { - Análise das ondas cerebrais } \\
\text { para compreender as } \\
\text { preferências (emoções) dos } \\
\text { consumidores quando estes são } \\
\text { expostos a determinadas imagens } \\
\text { de peças de roupas }\end{array}$ & $\begin{array}{c}\text { Dispositivos específicos para } \\
\text { medição das ondas cerebrais e } \\
\text { laboratório }\end{array}$ \\
\hline
\end{tabular}

Figura 10: Síntese da Pesquisa de Lü, Chen e Sui (2016). Fonte: as autoras.

A pesquisa de Hong et al. (2017) teve como objetivo propor um método de design colaborativo para projetar roupas personalizadas, para pessoas com escoliose. Primeiramente, o usuário é 
digitalizado por um scanner corporal 3D, que cria um avatar virtual traduzindo a forma morfológica do corpo com suas deformações físicas atípicas. Em seguida, ferramentas de desenho 3D e de desenvolvimento de moldes são utilizadas em conjunto entre o designer e o usuário, de modo a criar vestuários personalizados de acordo com as necessidades e preferências da pessoa com escoliose. A Figura 11 apresenta a síntese da pesquisa de Hong et al. (2017), informa o objetivo, o usuário pesquisado, as informações levantadas, e a técnica e/ou ferramentas utilizadas.

\begin{tabular}{|c|c|}
\hline \multicolumn{2}{|c|}{ Pesquisa de Hong et al. (2017) } \\
\hline Objetivo & Usuário Pesquisado \\
\hline $\begin{array}{c}\text { Propor um método de design } \\
\text { colaborativo para projetar roupas } \\
\text { personalizadas para pessoas com } \\
\text { escoliose }\end{array}$ & Pessoas com escoliose \\
\hline Informações Levantadas & Técnica/Ferramenta Utilizada \\
\hline $\begin{array}{c}\text { - Formas morfológicas do corpo } \\
\text { com suas deformações físicas } \\
\text { atípicas }\end{array}$ & $\begin{array}{l}\text { Medição Corporais com o uso do } \\
\text { Scanner corporal 3D }\end{array}$ \\
\hline $\begin{array}{c}\text { - Necessidades e preferências do } \\
\text { usuário com relação as suas } \\
\text { roupas }\end{array}$ & Design Colaborativo \\
\hline
\end{tabular}

Figura 11: Síntese da Pesquisa de Hong et al. (2017). Fonte: as autoras.

A pesquisa de Liu et al. (2018) teve como objetivo propor um método para gerar desenhos técnicos de vestuário de forma rápida e automática com base em medidas antropométricas, com o intuito de aprimorar a eficácia do design e reduzir os custos com o desenvolvimento de produtos. Para o desenvolvimento do software foi realizado um levantamento das medidas antropométricas, de 120 mulheres chinesas com idade entre 20 e 30 anos, por meio de um scanner corporal 3D. Entre as limitações do estudo destaca-se que os desenhos técnicos foram apenas de modelos de calças jeans, e que outros tipos de peças devem ser estudados. A Figura 12 apresenta a síntese da pesquisa de Liu et al. (2018), informa o objetivo, o usuário pesquisado, as informações levantadas, e a técnica e/ou ferramentas utilizadas.

\begin{tabular}{cc|}
\hline \multicolumn{2}{c}{ Pesquisa de Liu et al. (2018) } \\
\hline $\begin{array}{c}\text { Objetivo } \\
\text { Propor um método para gerar } \\
\text { desenhos técnicos de vestuário } \\
\text { de forma rápida e automática com } \\
\text { base em medidas } \\
\text { antropométricas }\end{array}$ & $\begin{array}{c}\text { Mulheres Chinesas } \\
\text { com idade entre } 20 \text { a } 30 \text { anos }\end{array}$ \\
\hline $\begin{array}{c}\text { Informações Levantadas } \\
\text { - Levantamento de Medidas } \\
\text { Antropométricas }\end{array}$ & \begin{tabular}{c} 
Técnica/Ferramenta Utilizada \\
Scanner Corporais com o uso do \\
\hline
\end{tabular} \\
\hline
\end{tabular}

Figura 12: Síntese da Pesquisa de Liu et al. (2018). Fonte: as autoras. 
A pesquisa de Nakayama e Martins (2018), refere-se ao resumo da dissertação da autora apresentada neste artigo como Nakayama (2016). A pesquisa apresenta assim, o mesmo objetivo, e os mesmos requisitos para o projeto de produtos de moda para pessoas com algum tipo de mobilidade reduzida.

A pesquisa de Liu et al. (2019) é uma continuidade da pesquisa de Liu et al. (2018), que teve como objetivo propor um método para desenvolvimento de modelagem de calça jeans com base nas dimensões do corpo. No software proposto, o usuário insere suas medidas corporais e seleciona um estilo de calça jeans, e então a modelagem é desenvolvida automaticamente. Caso necessário, é possível ajustar a modelagem no avatar virtual. A Figura 13 apresenta a síntese da pesquisa de Liu et al. (2019), informa o objetivo, o usuário pesquisado, as informações levantadas, e a técnica e/ou ferramentas utilizadas.

\begin{tabular}{|c|c|}
\hline \multicolumn{2}{|c|}{ Pesquisa de Liu et al. (2019) } \\
\hline Objetivo & Usuário Pesquisado \\
\hline $\begin{array}{l}\text { Propor um método para } \\
\text { desenvolvimento de modelagens } \\
\text { de calça jeans com base nas } \\
\text { dimensões do corpo }\end{array}$ & $\begin{array}{l}\text { Mulheres Chinesas } \\
\text { com idade entre } 20 \text { a } 30 \text { anos }\end{array}$ \\
\hline Informações Levantadas & Técnica/Ferramenta Utilizada \\
\hline $\begin{array}{c}\text { - Levantamento de Medidas } \\
\text { Antropométricas }\end{array}$ & Medição Corporais \\
\hline
\end{tabular}

Figura 13: Síntese da Pesquisa de Liu et al. (2018). Fonte: as autoras.

A pesquisa de Townsend et al (2019) teve como objetivo desenvolver um modelo de negócios com base na compreensão das necessidades e expectativas das consumidoras mais velhas em relação à moda. Para isso, realizou-se uma coleta de dados com 45 inglesas com mais de 55 anos, envolvendo a realização de entrevistas, oficinas, medições corporais e posterior confecção de corpetes para prova. Após análise dos dados, os autores verificaram: 1) limitações da oferta: constatou-se sentimento de insatisfação e desconexão com a moda que é oferecida a estas mulheres; 2) inadequação dos tamanhos: constatou-se que os corpetes desenvolvidos e provados de acordo com as medidas individuais não possuem relação com os tamanhos rotulados nas peças vendidas no mercado.

$\mathrm{Na}$ sequência, as participantes da pesquisa trabalharam em conjunto com os autores/pesquisadores para desenvolver uma coleção com 30 looks que respondesse às questões práticas, emocionais e éticas levantadas. Como conclusão, a pesquisa apresenta o design colaborativo como uma estratégia que fortalece o relacionamento entre o cliente e o designer, permitindo que ambos compreendam conjuntamente as necessidades dos consumidores. (TOWNSEND et al, 2019). A Figura 14 apresenta a síntese da pesquisa de Townsend et al (2019), informa o objetivo, o usuário pesquisado, as informações levantadas, e a técnica e/ou ferramentas utilizadas. 


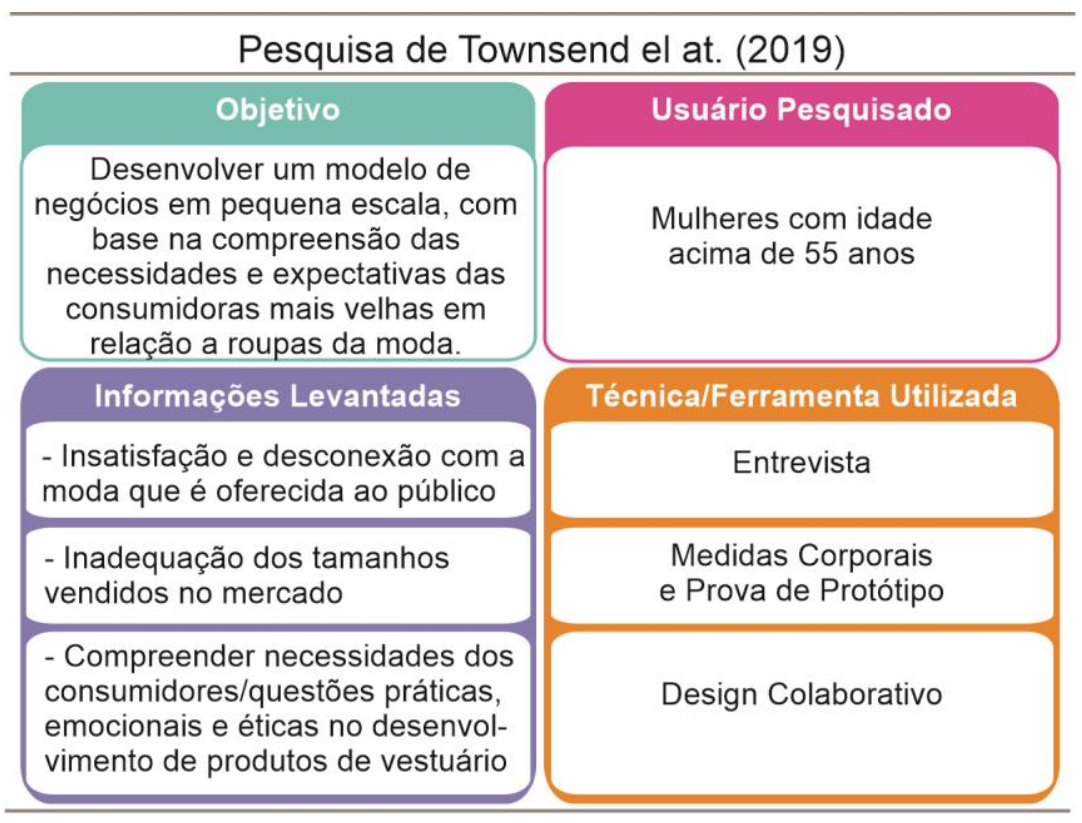

Figura 14: Síntese da Pesquisa de Townsend (2019). Fonte: as autoras.

\section{Resultado e Discussão}

Nesta seção, apresentam-se os resultados e discussões relacionados às 13 pesquisas selecionadas na revisão sistemática, de acordo com os tópicos: 1) objetivo pelo qual as pesquisas realizaram a coleta de dados sobre o usuário; 2) identificação do modo de coleta de dados sobre o usuário e a recorrência; 3) identificação da coleta de dados com a fase projetual.

Quanto à identificação do objetivo pelo qual as pesquisas realizaram a coleta de dados sobre o usuário de vestuário, temos: para desenvolver uma ferramenta, desenvolver e aplicar um método, desenvolver um produto de vestuário, e desenvolver um modelo de negócios na área de confecção de vestuário.

- Para desenvolver uma ferramenta (3 pesquisas): Nakayama (2016) e Nakayama e Martins (2018) - ferramenta metodológica para criação de produtos de moda para pessoas com mobilidade reduzida; Leet el al (2015) - ferramenta de mineração das informações de banco de dados para auxiliar na compreensão das preferências dos consumidores.

- Para desenvolver e aplicar um método (7 pesquisas): Collet (2016) - método para criação de produto de moda inclusivo; Souza (2016) - método para desenvolver vestuário adaptado para cadeirantes; Brogin (2019) - método para desenvolver moda funcional para pessoas com deficiência motora; Lü, Chen e Sui (2016) - método para análise psicológica dos consumidores; Hong et al (2017) - método de design colaborativo para projetar roupas para pessoas com escoliose; Liu et al (2018) - método para desenhos técnicos com base nas medidas antropométricas; Liu et al (2019) - método para desenvolvimento de modelagens com base nas medidas antropométricas.

- Para desenvolver um produto de vestuário (2 pesquisas): Mentone (2018) - protótipo para gestantes; Tse e Chan (2016) - coleção de moda festa para gestantes. 
- Para desenvolver um modelo de negócios na área de confecção de vestuário (1 pesquisa): Townsend et al (2019) - modelo de negócios para consumidoras com idade acima de 55 anos.

Observa-se que 9 pesquisas propõem o desenvolvimento de um método ou ferramenta para auxiliar no desenvolvimento de produtos de vestuário para usuários específicos, sendo 6 pesquisas com foco no desenvolvimento de produtos para pessoas com deficiência (mobilidade reduzida, cadeirante, estomizados, escoliose), 2 pesquisas com foco em mulheres gestantes, e 1 pesquisa com foco para mulheres com idade acima dos 55 anos. Assim, estas pesquisas que foram desenvolvidas nos últimos anos (2015 a 2019) surgem para complementar os poucos métodos da área do Design de Moda com abordagem centrada no usuário.

Na sequência, identificou-se como cada uma das 13 pesquisas realizou a coleta de dados (utilização de técnicas, métodos, ferramentas, equipamentos etc.) sobre o usuário do produto de vestuário, e a recorrência. Como resultado, tem-se 10 modos diferentes: 1) Pesquisa Bibliográfica (3 pesquisas); 2) Análise de produtos concorrentes ou similares (2 pesquisas); 3) Entrevista (3 pesquisas); 4) Questionário (2 pesquisas); 5) Análise de Tarefa (1 pesquisa); 6) Design Colaborativo (3 pesquisas); 7) Medidas Corporais (3 pesquisas); 8) Teste de Usabilidade (3 pesquisas); 9) Pesquisa Psicológica (1 pesquisa); 10) Pesquisa em Banco de Dados (1 pesquisa) (Figura 15).

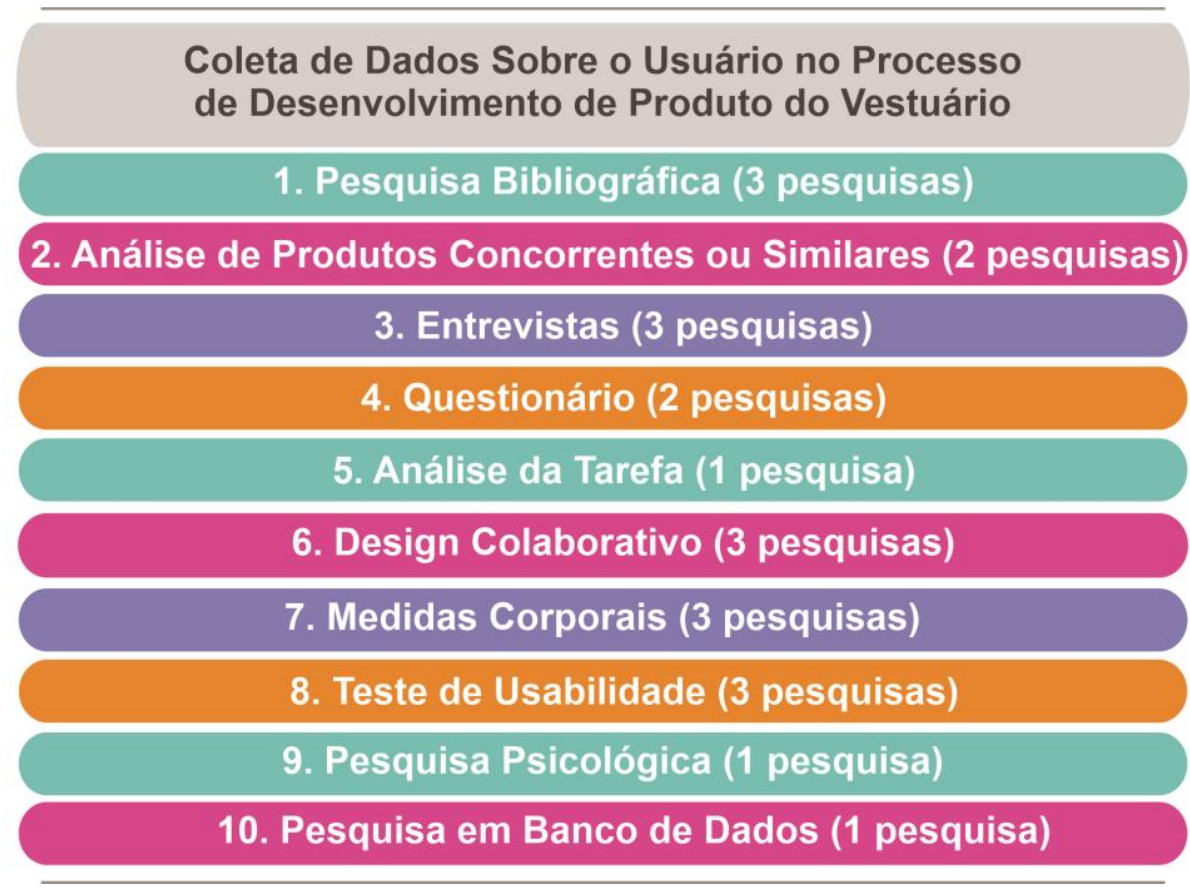

Figura 15 - Coleta de Dados sobre o Usuário no Processo de Desenvolvimento de Produto do Vestuário. Fonte: as autoras.

Observa-se que os 8 primeiros modos utilizados para a coleta de dados sobre o usuário podem ser facilmente executados no decorrer do desenvolvimento de produto do vestuário, seja em ambiente acadêmico ou nas empresas de confecção. A exceção, são os 2 últimos modos de coleta de dados que apresentam uma execução mais complexa, a pesquisa psicológica e pesquisa em banco de dados, porque exigem respectivamente, laboratório com dispositivos específicos e 
ambiente controlado, e o desenvolvimento de tecnologia específica para analisar o banco de dados.

Com relação ao uso da pesquisa bibliográfica, além da fácil execução, se apresenta como um modo que evita a exposição de usuários a situações desnecessárias ou a criação de expectativas. Especialmente nos casos em que há a necessidade de coletar dados junto a usuários com deficiência, como nas pesquisas de Collet (2016) - mulheres estomizadas, e Nakayama (2016) usuários com mobilidade reduzida.

A análise de produtos concorrentes ou similares, realizadas por Tse e Chan (2016) a partir de uma pesquisa de imagens na internet, a fim de verificar situações de constrangimento ocasionados pelo uso das vestimentas de gestantes, colabora com os autores no desenvolvimento da pesquisa, pois caso optassem por verificar essas situações de constrangimento de outra maneira, como por exemplo, em uma análise de tarefa, seria necessário encontrar gestantes e acompanhá-las nas atividades de vestir e despir, e na rotina diária. Com isso, observa-se a necessidade de ponderar, no decorrer do projeto, o tempo dedicado à realização da coleta de dados, porque alguns modos podem exigir mais dedicação do que outros, bem como o fácil acesso e o contato com o usuário pesquisado.

Quanto às entrevistas e questionários, possivelmente manifestam-se como a primeira opção quando se pretende realizar coleta de dados diretamente com o usuário, pois somadas apresentam a maior recorrência entre os modos utilizados (5 pesquisas). As pesquisas de Souza (2016) e Brogin (2019) que se utilizam de questionários para a coleta de dados com usuários, lembram de adicionar como respondentes os profissionais da saúde (terapeutas ocupacionais, fisioterapeutas) e cuidadores (mãe, pai, irmão, e outros familiares), já que essas pessoas quando auxiliam no vestir e despir, também reconhecem certas necessidades específicas que podem colaborar no processo de desenvolvimento do produto de vestuário.

O levantamento de medidas corporais pode ser realizado com o uso das fitas métricas ou de scanner corporal 3D, que garante maior fidedignidade, como propõem as pesquisas de Liu et al. (2018) e Hong et al. (2017). Observa-se a escolha adequada do uso do scanner corporal 3D, com relação ao usuário pesquisado por Hong et al. (2017), que são as pessoas com escoliose, para as quais o scanner cria um avatar virtual de acordo com a forma morfológica, com suas deformações físicas típicas da escoliose. Destaca-se que ao tomar as medidas com a fita métrica, deve-se analisar as limitações e a forma morfológica do usuário pesquisado, porque são necessárias as medidas referentes a posição estática mais frequente, as medidas de relaxamento ou tensionamento corpóreo, e as dos movimentos constantes (NAKAYAMA, 2016). Como por exemplo, para desenvolver um vestuário para um usuário cadeirante, é necessário tomar-lhe as medidas das posições estáticas mais frequentes, ou seja, sentado e deitado (BROGIN, 2019).

Em suma, como o produto de vestuário está em contato direto com o usuário é indispensável a tomada de medidas corporais para o desenvolvimento da modelagem e posterior confecção. Entretanto, a tomada de medidas corporais revela informações adicionais, como a pesquisa de Townsend et al (2019) que teve como objetivo desenvolver um modelo de negócios, e a tomada de medidas revelou inadequação dos tamanhos vendidos no mercado quando comparado a diferentes marcas, o que gera sentimento de confusão e frustração nas usuárias pesquisadas. 
Quanto aos testes de usabilidade, destaca-se que devem ser realizados em usuário cujas medidas corporais estejam de acordo com as coletadas para o desenvolvimento da modelagem. Caso contrário, os ajustes no vestuário serão realizados em cima de medidas corporais não correspondentes, o que pode invalidar o teste de usabilidade.

Por fim, identificou-se o modo utilizado para a coleta de dados com a fase projetual, que foram denominadas de forma genérica, como as fases de planejamento, criação, modelagem e prototipagem (Figura 16).

\begin{tabular}{|c|c|c|c|c|}
\hline & & Fases Projetuais do [ & Jesign de Moda & \\
\hline Coleta de Dados & Planejamento & Criação & Modelagem & Prototipagem \\
\hline Pesquisa Bibliográfica & $\begin{array}{c}\text { Collet (2016) } \\
\text { Nakayama (2016) } \\
\text { Nakayama e Martins (2018) }\end{array}$ & & & \\
\hline $\begin{array}{l}\text { Análise de Produtos } \\
\text { Concorrentes/Similares }\end{array}$ & $\begin{array}{l}\text { Tse e Chan (2016) } \\
\text { Brogin (2019) }\end{array}$ & & & \\
\hline Entrevistas & $\begin{array}{c}\text { Tse e Chan (2016) } \\
\text { Mentone (2018) } \\
\text { Townsend et al (2019) }\end{array}$ & & & \\
\hline Questionário & $\begin{array}{l}\text { Souza (2016) } \\
\text { Brogin (2019) }\end{array}$ & & & \\
\hline Análise da Tarefa & Brogin (2019) & & & \\
\hline Design Colaborativo & & $\begin{array}{l}\text { whsend et al (2019) } \\
\text { Hong et al (2017) } \\
\text { Brogin (2019) }\end{array}$ & & \\
\hline Medidas Corporais & Townsend et al (2019) & Liu et al (2018) & $\begin{array}{l}\text { Hong et al (2017) } \\
\text { Liu et al (2019) } \\
\text { Brogin (2019) }\end{array}$ & \\
\hline Teste de Usabilidade & & & & $\begin{array}{c}\text { Souza (2016) } \\
\text { Mentone }(2018) \\
\text { Brogin }(2019)\end{array}$ \\
\hline Pesquisa Psicológica & Lü, Chen e Sui (2016) & & & \\
\hline Pesquisa em Banco de Dados & Lee et al (2015) & & & \\
\hline
\end{tabular}

Figura 16 - Relação entre a Coleta de Dados e a Fase Projetual do Design de Moda. Fonte: as autoras.

Observa-se que a coleta de dados sobre o usuário ocorre com maior frequência na fase de planejamento do projeto, e que nas demais fases do projeto (criação, modelagem e prototipagem) os modos utilizados para a coleta de dados junto ao usuário decrescem. Neste contexto, cabe destacar que "o desafio está não somente em levantar informações, analisá-las e chegar a solução para problemas existentes, mas sim em testar, avaliar e validar produtos ou serviços planejados para um mundo real, para usuários reais" (MERINO, 2016, P.7).

\section{Conclusão}

Este artigo aborda o processo de desenvolvimento de produto de moda que requer a realização de diferentes pesquisas a respeito do produto, do contexto de uso e do usuário. Porém, entre elas, acredita-se que a coleta de dados sobre o usuário, é desconsiderada ou pouco explorada com ferramentas e métodos, nos projetos da área do Design de Moda. Assim, este artigo buscou identificar as técnicas e ferramentas utilizadas na coleta de dados sobre o usuário no processo de desenvolvimento de produtos de vestuário. 
Para tal, realizou-se uma revisão sistemática da literatura, na qual foram selecionadas 13 pesquisas. Como resultado, apresenta-se 10 modos para a coleta de dados sobre o usuário no decorrer do processo de desenvolvimento de produtos de vestuário: 1) Pesquisa Bibliográfica; 2) Análise de produtos concorrentes ou similares; 3) Entrevista; 4) Questionário; 5) Análise de Tarefa; 6) Design Colaborativo; 7) Medidas Corporais; 8) Teste de Usabilidade; 9) Pesquisa Psicológica; e 10) Pesquisa em Banco de Dados. Percebeu-se que a maioria destes modos são utilizados com maior frequência na fase de planejamento do projeto, e que para as demais fases a realização de coleta de dados sobre o usuário decresce. Assim, cabe enfatizar, que a coleta de dados sobre o usuário deve ser priorizada, e considerada em todo o processo de desenvolvimento do vestuário, de modo a desenvolver produtos adequados aos mesmos.

Percebeu-se o aparecimento de pesquisas recentes, nos últimos 6 anos, que tiveram como objetivo propor métodos e ferramentas para auxiliar no desenvolvimento de produtos de vestuário para um usuário específico, e vem justamente para complementar os poucos métodos existentes na área do Design de Moda que apresentam abordagem centrada no usuário. Entre elas, observase o predomínio de pesquisas voltadas para o desenvolvimento de produtos de vestuário para pessoas com deficiência (mobilidade reduzida, cadeirante, estomizados, escoliose). Acredita-se que mesmo sendo desenvolvidas para um usuário específico, a pesquisa pode contribuir com a coleta de dados e desenvolvimento de produtos para usuários de modo geral.

Como contribuição científica e social, acredita-se que a partir do momento em que se obtém este conhecimento sobre o modo de realizar a coleta de dados, a fase projetual, e a especificidade do usuário pesquisado, é possível aprofundar e estimular a realização de coleta de dados nos métodos de projeto de moda, seja no ambiente acadêmico ou empresarial. Como estudos futuros, sugere-se verificar quais devem ser os dados a serem coletados a respeito do usuário, a fim de gerar ferramentas que o considere no decorrer de todo o processo de desenvolvimento de produto do vestuário.

\section{Referências}

BACK, Nelson et al. Projeto integrado de produtos: planejamento, concepção e modelagem. Barueri - São Paulo: Manole, 2008.

BROGIN, Bruna. Método de Design para Cocriação de Moda Funcional para Pessoas com Deficiência. 2019. 411 f. Tese (Doutorado) - Curso de Design, Artes, Comunicação e Design, Universidade Federal do Paraná, Curitiba, 2019.

COLLET, Jessica Andressa. Método para a moda complementado por metodologias de design de produto: Aplicação no desenvolvimento de produtos de moda praia para estomizadas. 2016. 256 f. Dissertação (Mestrado) - Curso de Design, Universidade Federal do Rio Grande do Sul, Porto Alegre, 2016.

FERENHOF, Helio Aisenberg; FERNANDES, Roberto Fabiano. Passos para construção da Revisão Sistemática e Bibliometria. V. 2.03 Disponível em: <http://www.igci.com.br/artigos/passos_rsb.pdf>.

HONG, Yan et al. Virtual reality-based collaborative design method for designing customized garment for disabled people with scoliosis. International Journal Of Clothing Science And Technology, [s.1.], v. 29, n. 2, p.226-237, 18 abr. 2017. Emerald. http://dx.doi.org/10.1108/ijcst-07-2016-0077. 
LEE, Carmen Kar Hang et al. Fuzzy association rule mining for fashion product development. Industrial Management \& Data Systems, [s.1.], v. 115, n. 2, p.383-399, 9 mar. 2015. Emerald. http://dx.doi.org/10.1108/imds-09-2014-0277.

LIU, Kaixuan et al. Parametric design of garment flat based on body dimension. International Journal Of Industrial Ergonomics, [s.1.], v. 65, p.46-59, maio 2018. Elsevier BV. http://dx.doi.org/10.1016/j.ergon.2018.01.013.

LIU, Kaixuan et al. Parametric design of garment pattern based on body dimensions. International Journal Of Industrial Ergonomics, [s.1.], v. 72, p.212-221, jul. 2019. Elsevier BV. http://dx.doi.org/10.1016/j.ergon.2019.05.012.

LÜ, Jia; CHEN, Dongsheng; SUI, Yue. Event-related potentials technique using in affective fashion design. International Journal Of Clothing Science And Technology, [s.1.], v. 28, n. 1, p.77-91, 7 mar. 2016. Emerald. http://dx.doi.org/10.1108/ijcst-09-2014-0110.

MENTONE, Daniela Antunes Nolasco. Proposta de desenvolvimento de produtos de malha para gestantes utilizando a tecnologia seamless. 2018. 27 f. Dissertação (Mestrado) - Curso de Têxtil e Moda, Universidade de São Paulo, São Paulo, 2018.

MERINO, Giselle Schmidt Alves Díaz. GODP - Guia de Orientação para Desenvolvimento de Projetos: Uma metodologia de Design Centrado no Usuário. Florianópolis: Ngd/Ufsc, 2016. Disponível em: 〈www.ngd.ufsc.br〉. Acesso em: 12 jul. 2020.

NAKAYAMA, Gabriela Yoshie. Desenvolvimento de produtos de moda para pessoas com mobilidade reduzida: ferramenta metodológica pautada na ergonomia. 2016. $112 \mathrm{f}$. Dissertação (Mestrado) - Curso de Design, Universidade Federal de Pernambuco, Recife, 2016.

NAKAYAMA, Gabriela Yoshie; MARTINS, Laura Bezerra.. Fashion Design Methodology Tools in Products' Development for People with Disabilities and Low Mobility. Advances In Intelligent Systems And Computing, [s.1.], p.1699-1704, 11 ago. 2018. Springer International Publishing. http://dx.doi.org/10.1007/978-3-319-96071-5 174.

SANCHES, Maria Celeste de Fátima. Projetando moda: diretrizes para a concepção de produtos. In: PIRES, D.B. Design de Moda: olhares diversos. Barueri: Editora Estação das Letras e Cores, p. 289-302, 2008

SOUZA, Letícia Nascimento de. Proposta de metodologia para adaptação de vestuário para pessoas com deficiência física (cadeirante). 2016. 22 f. Dissertação (Mestrado) Curso de Têxtil e Moda, Universidade de São Paulo, São Paulo, 2016.

TOWNSEND, Katherine et al. Fashioning clothing with and for mature women: a smallscale sustainable design business model. Management Decision, [s.1.], v. 57, n. 1, p.3-20, 14 jan. 2019. Emerald. http://dx.doi.org/10.1108/md-12-2016-0942.

TSE, Florence T.F.; CHAN, Catherine Y.P.. New approach for fashion design. Research Journal Of Textile And Apparel, [S.L.], v. 20, n. 1, p. 53-60, 14 mar. 2016. Emerald. http://dx.doi.org/10.1108/rjta-02-2015-0004.

\section{Sobre o autor}

\section{Elen Makara}

Professora do Instituto Federal de Educação, Ciência e Tecnologia de Santa Catarina - Campus Gaspar. Doutoranda em Design na linha de Gestão na Universidade Federal de Santa Catarina (UFSC). Mestre em Design pela Universidade Federal de Santa Catarina (UFSC). Bacharel em Moda com habilitação em Estilismo pela Universidade do Estado de Santa Catarina (UDESC). https://orcid.org/0000-0001-9496-1862 


\section{Giselle S. A. D. Merino}

Pesquisadora CNPQ - PQ 1D. Profa. Universidade Federal de Santa Catarina (Pós-graduação) e Universidade do Estado de Santa Catarina (Graduação e Pós-graduação). Pós-doutorado em Fatores Humanos pela Universidade do Estado de Santa Catarina/ Estágio na Universidad Politécnica de Valencia (Espanha). Dra. em Engenharia de Produção (linha: Produto e Processo metodologias de projeto de Design) pela Universidade Federal de Santa Catarina (UFSC). https://orcid.org/0000-0003-4085-3561 